\title{
Isolation and Identification of the Pathogens Causing Cycas panzhihuaensis Stem Rot Disease
}

\author{
Yi Zheng', Zhi-xiang $\mathbf{Y u}^{2}$, Bin $\mathrm{Wu}^{1}$ \\ ${ }^{1}$ School of Biology and Chemistry Engineering, Panzhihua University, Panzhihua City, Sichuan 617 000, P. R. China \\ ${ }^{2}$ Administration Bureau of Cycas panzhihuaensis National Nature Reserve, Panzhihua City, Sichuan 617 000, P. R. China
}

\begin{abstract}
The objective of the research is to explore the pathogenesis of the C. panzhihuaensis stem rot disease as well as to find out the prevention and treatment of it. Sick tissue with typical symptoms of the stem rot disease was collected in C. panzhihuaensis National Nature Reserve. Pathogens were isolated using the routine tissue isolation method, and the pathogenicity study was carried out. In addition, routine morphological identification and rDNA ITS sequence analysis were also applied to the strains with pathogenicity. The results showed that among the four strains isolated from the ill-healthy tissue, the infection rate of Cym001 reached $76.3 \% 15$ days after re-inoculation. Hence, Cym001 was determined to be the pathogen causing the C. panzhihuaensis stem rot disease and was then identified to be Fusarium solani. The present research provides a theoretical basis for the prevention and control of the $C$. panzhihuaensis stem rot disease.
\end{abstract}

Keywords: Cycas panzhihuaensis; pathogen; identification; ITS sequence; Fusarium solani.

\section{Introduction}

Cycas panzhihuaensis (L. Zhou et al., Y. Yang) is a new species of the ancient relic plant of Cycas, Cycadaceae plants that was first discovered in 1971 and officially given the name in 1981 (L. Zhou, 1981). The wild C. panzhihuaensis grows in large patches in the dry-hot river valley of Jinshajiang, and is on the list of the Grade 1 National Protected Plants of China. Over half of the wild Cycas resources in China are grown in the $C$. panzhihuaensis National Nature Reserve which was officially established in western Panzhihua in 1996; they are the highest in altitude, largest in area, biggest in number and most concentrated in distribution of the wild cycads worldwide (Y.Q. Hao et al., 2011). Common diseases that strike the Cycadaceae plants are anthracnose, speck, sooty blotch, Fusarium stem rot and leaf blight et al. (M.Zh. Fu et al., 2005; X.X. Li, 2014). There was no report on the diseases of the C. panzhihuaensis in the past, but in recent years, the routine monitoring of the National Nature Reserve revealed that a small amount of the plants were suspected to die due to the stem rot disease. Our research made a preliminary investigation on the diseases that stroke the C. panzhihuaensis. We isolated and identified the pathogens and carried out the pathogenicity study to provide scientific evidence for the control of the $C$. panzhihuaensis stem rot disease.

\section{Host animals of HEV}

\subsection{Materials}

\subsubsection{Biological Materials}

The scale stems of the C. panzhihuaensis were collected in the C. panzhihuaensis National Nature Reserve $\left(26^{\circ} 62^{\prime} \mathrm{N}\right.$, 10159’E) in western Panzhihua City, Sichuan Province, P. R. China, from July, 2013 to August, 2015.

\subsubsection{Key Instruments and Agents}

Key Instruments: autoclave, bio-chemical incubator, superclean bench, micropipette, constant temperature and humidity incubator, low-temperature refrigerator, digital microscope, horizontal electrophoresis chamber, transilluminator, high-speed refrigerated centrifuge, table-top centrifuge, PCR instrument, et al..

Key Agents: sodium hypochlorite, absolute ethanol, glucose, agar, EDTA-Na, Tris, Ethidium Bromide, Taq enzyme, dNTP, Marker, primer, et al..

\subsection{Methods}

2.2.1 Investigation on the disease characteristics and the diseased plants.

A certain area in the National Protected Reserve was chosen as the sample, and diseased plants were investigated in the four seasons respectively. Characteristics of the diseased plants were observed, the amount and the onset time of the diseased plants were analyzed statistically, and the distance between the diseased plants were estimated by eye.

\subsubsection{Isolation of the pathogen.}

The isolation and culture of the tissue were carried out on the PDA medium using routine methods (Y.Li et al., 2013). The surface of the scale stems of C. panzhihuaensis with typical symptoms was first disinfected with $70 \%$ alcohol cotton ball, and then sterilized with newly prepared 3\% sodium hypochlorite for 30 seconds, and then washed with sterile water for 3 times. Five plant tissues were isolated from each of the chosen junction between the ill and healthy tissues, and were placed into the same culture dish and were incubated in the biochemical incubator at $25^{\circ} \mathrm{C}$. In this study, 30 repetitions were designed (10 repetitions for each isolation and 3 isolations in total), and the healthy scale stems were used as control. The isolated tissues were cultured for 4 to 15 


\section{International Journal of Science and Research (IJSR) \\ ISSN (Online): 2319-7064 \\ Index Copernicus Value (2013): 6.14 | Impact Factor (2014): 5.611}

days for observation, and the mycelium grown from the culture were collected, numbered, purified, classified and analyzed statistically. Effective strains were preserved on the PDA slant culture medium in the refrigerator at $4^{\circ} \mathrm{C}$.

\subsubsection{Pathogenicity Study}

The isolated suspected pathogens were cultured in the PDA medium for 7 days, using the spore infection method (J.J. Luo et al., 2014). Sterile water was then added to the culture to make the spore suspension, with the spore concentration being 40-60 per microscopic field (400X). Plant infection experiment was carried out using fresh and healthy $\mathrm{C}$. panzhihuaensis scale stems. The surface of the scale stems was disinfected using 3\% sodium hypochlorite solution and then washed 3 times with sterile water. Sterile filter paper with a diameter of $0.5 \mathrm{~cm}$ was dipped into the spore suspension and then lightly placed onto the surface of the scale stems. The scale stems were divided into the injured and non-injured groups. In the injured group, the surface of the scale stems was stabbed with a sterile syringe needle to induce an injured inoculation. 30 repetitions were designed (10 repetitions for each isolation and 3 isolations in total), and the sterile water was used as control (ck). The inoculated scale stems were cultured in the bio-chemical incubator at $25^{\circ} \mathrm{C}$ for 3 to 15 days, and the incidence was observed and recorded everyday. Pathogens were re-isolated and observed based on Koch's Postulates using the method mentioned in 1.2.2.

\subsubsection{Observation of the pathogen morphology and preliminary identification}

The morphology of the purified pathogens on the PDA plate culture dish as well as the shape and size of the conidiospores on the insert culture dish were observed. Preliminary identification was carried out by comparing the above characteristics observed in our research with the ones mentioned in the literature (J.Ch. Wei, 1979; H.L. Barnett et al., 1977).

\subsubsection{Molecular biology identification of the pathogens.}

The 5.8s rDNA-ITS sequence was used for the molecular identification of the pathogens. The mycelium of the pathogens were inoculated into the liquid PDA. The mycelium pellet from the culture was ground with liquid nitrogen, and the total DNA of the pathogens was extracted using the CTAB method (L.Y. Yang et al., 2006). Fungal rDNA general primer ITS1 and ITS4 were used for the PCR amplification. The ITS1 sequence was 5'TCCGTAGGTGAACCTGCGG -3', and the ITS4 sequence was 5'-TCCTCCGCT TATTGATATGC-3'. The PCR reaction system was $20 \mu \mathrm{L}$, and the PCR reaction conditions were: pre-denaturation at $95^{\circ} \mathrm{C}$ for $5 \mathrm{~min}$, repeated once; denaturation at $95^{\circ} \mathrm{C}$ for 30 seconds, annealing at $55^{\circ} \mathrm{C}$ for 30 seconds, and elongation at $72^{\circ} \mathrm{C}$ for 40 seconds, repeated 35 times; final elongation at $72^{\circ} \mathrm{C}$ for $10 \mathrm{~min}$. After the PCR products were examined withh $1 \%$ agar-gel electrophoresis, they were purified by Shanghai Meiji Company and sequenced by the ABI3730XL sequencer. The sequences were assembled and submitted to GenBank, and then were compared with the database using a Blast search. MEGA
(4.0) was used to establish the phylogenetic tree for the acquired homologous sequence, so as to identify the taxonomic status of the pathogens.

\section{Results and Analysis}

\subsection{Characteristics of the C. panzhihuaensis stem rot disease}

Characteristics of the C. panzhihuaensis stem rot disease were shown in Fig. 1. In the early stage of the disease, the pinnate leaves turned yellow or green wilted. And with the progression of the disease, the middle or lower-middle part of the plant stems started to rot and soften, resulting in the lodging and death of the plants. Anatomy of the diseased plant stems showed the pith of the plant stems turned brown and rotted. The scale stems turned brown spreading from the pith due to the infection, and obvious rotten spots could be observed (Fig. 2). After 2 years investigation, we noticed that the amount of the plants infected with the disease was rather low (lower than $0.1 \%$ of the number of the total mature plants within the sample area), that the symptoms occurred more often during August to October which were the season of high temperature and humidity locally, and that the distance between diseased plants were not close (estimated distance by eye was over 25m). Judging from these characteristics, this stem rot disease is supposed to be caused by a certain pathogen, which is not epidemic and does not cause much damage.

\subsection{Result of the pathogen isolation}

The purified pathogens can be classified into four strains that were numbered Cmy001、Cmy003、Cmy08、Cmy011, with a percentage of $87.5 \%, 6.3 \%, 3.6 \%, 2.7 \%$, respectively. Among these strains, Cmy001 was the obvious advantageous strain. Pathogens could also be obtained from healthy scale stems, but they grew slowly and there was no obvious advantageous strain. Therefore, these pathogens were suspected to be the endophytic fungi of the C. panzhihuaensis, and no further studies were carried out.

\subsection{Pathogenicity Experiment}

No obvious symptoms were observed after the strain Cmy003, Cmy08, Cmy011 were inoculated to the injured or the non-injured plants. The incidence was lower than 5\%, which was close to the incidence of the sterile water control group, indicating these strains were not the pathogens causing the disease. Obvious symptoms could be observed five days after the strain Cmy001 was inoculated to the injured group, and the 7-day and 15-day incidences reached $23.3 \%$ and $76.3 \%$, respectively. Rotting spots were oval and dark brown, and the symptoms were similar to those observed in the stem rot disease of the C. panzhihuaensis from the National Natural Reserve (Fig. 3). From the junction between the ill and healthy tissues, strains that resembled the ones inoculated into the tissues, in terms of the colony characteristics and the morphology of the conidiospores, could be isolated. This demonstrated that Cmy001 was the pathogen causing the $\mathrm{C}$. panzhihuaensis 


\section{International Journal of Science and Research (IJSR) \\ ISSN (Online): 2319-7064 \\ Index Copernicus Value (2013): 6.14 | Impact Factor (2014): 5.611}

stem rot disease. No obvious symptoms were observed in the non-injured group. This could be due to the tight epidermal tissue of the scale stems which made it difficult for the pathogen spores to invade into the inner tissue.

\subsection{Morphology identification of the pathogens.}

The strain Cmy001 was isolated and purified, and its colony and microscopic characteristics were shown in Fig. 4. Five days after cultured in the PDA medium, the colony was white on the upper surface and purple blue on the back. The mycelium was white and septate, and conidiophores could be observed. Large conidiospores were formed within the pionnotes, and then spread between the mycelium. Spores were initially smooth, spindle or oval, with a diameter of
5.5 9.5×27.5 38um. They then became sickle-shaped with 3-4 septum after a long time. This morphology falls into the Fusarium species (Fusarium sp.).

\subsection{The ITS sequence analysis result}

The ITS region of the strain Cmy001 was amplified to 557 bp. NCBI comparison (Fig. 5) showed that Cmy001 (KT441029) was 100\% homologous to the Fusarium solani strain (FJ224382.1), F. solani NBRC 8505 (AB674260.1), F. solani isolate T70 (FJ459978.1) in the GenBank. Therefore, Cmy001 could be confirmed to be F. soloni , and could be identified for the moment as the $C$. panzhihuaensis specialized form of F. solani.
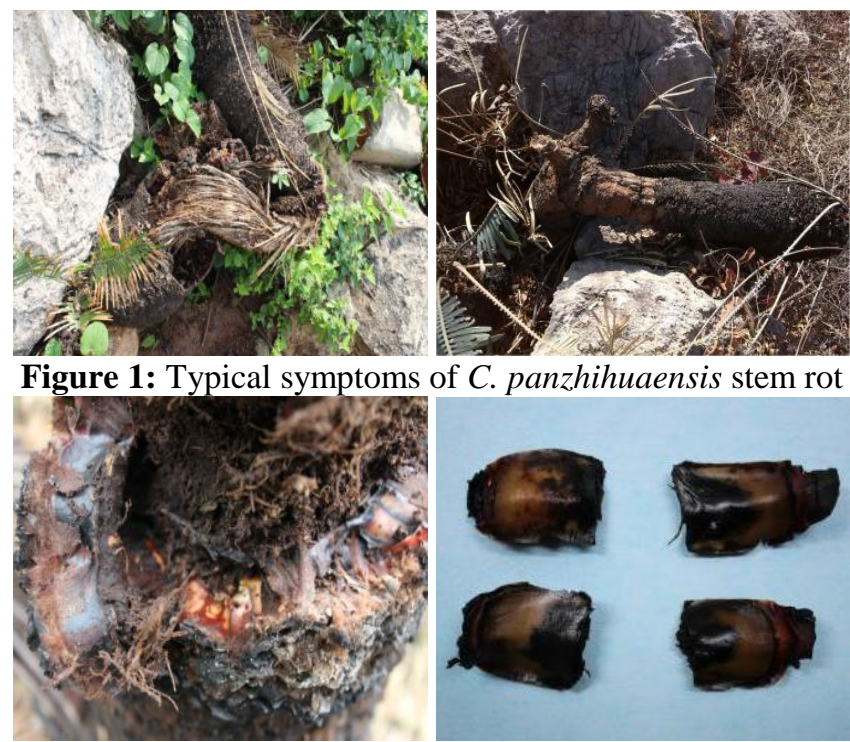

Figure 2: Bulblet tissue of C. panzhihuaensis stem rot

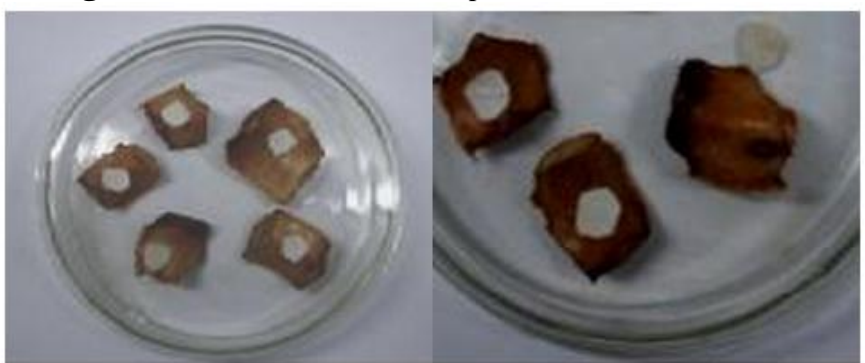

Figure 3: Symptom on artificially inoculated bulblet of $C$. panzhihuaensis
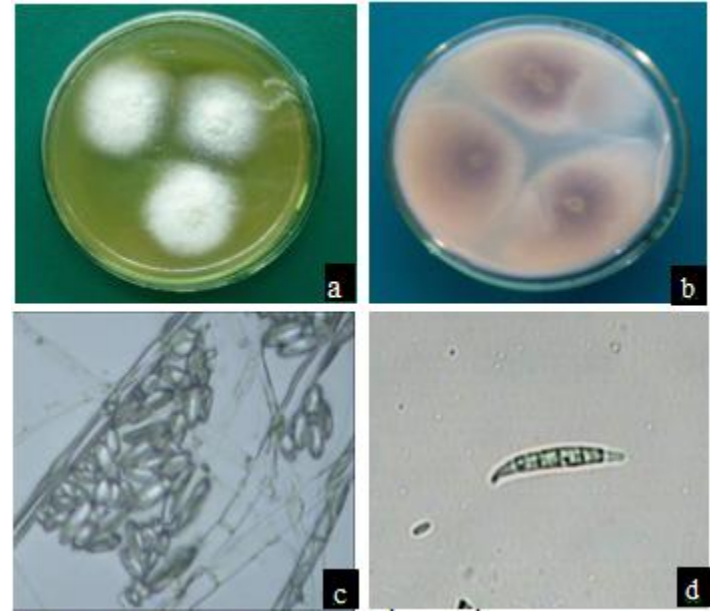

Figure 4: Colony culture characteristics of strain Cmy001 and micro-morphological characteristics of conidium

(a. colony obverse $3 \mathrm{~d} 25^{\circ} \mathrm{C}$ b. reverse $5 \mathrm{~d} 25^{\circ} \mathrm{C}$ c. mycelium d.spore)

\section{Volume 5 Issue 3, March 2016}




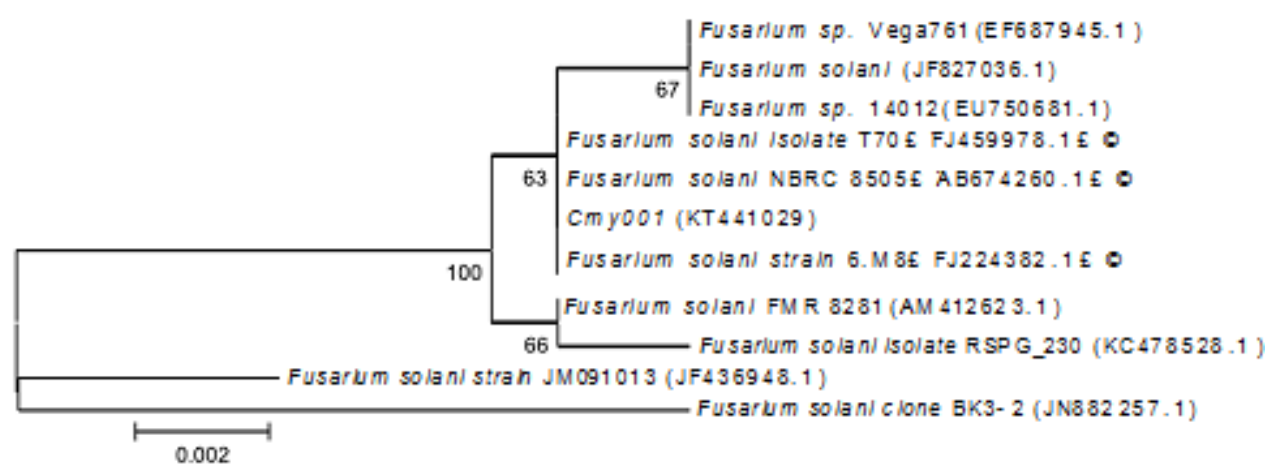

Figure 5: Tree analysis on the comparison of the strain Cmy001 5.8s rDNA ITS Sequence Homology

\section{Discussion and Conclusion}

From the investigation of the characteristics of the C.panzhihuaensis stem rot disease, the isolation of the pathogens, the pathogenicity studies and the identification of the pathogens, it could be demonstrated that the pathogen causing the C. panzhihuaensis stem rot disease is F. solani, which belongs to the Imperfecti fungi, Moniliales, Tuberculariaceae , Fusarium sp., and is a plant pathogenicfungus, which causes wilting diseases and root rot diseases in many grain crops and cash crops. Fusarium solani was reported to be the major pathogen causing root rot diseases in jatropha curcas, Pueraria thomsonii and Rhizoma Dioscoreae (Y.K. Wu et al., 2011; L.W. Lin et al., 2003; L.T. Shen et al., 2012). F. solani has a wide distribution, and is a soilinhabitant fungus that could carry out saprogenesis in soil for a long time and cause damage to its symbiotic host. The infection of $\mathrm{F}$. solani to its host and its pathogenicity mechanisms have been reported (D.F. Bateman et al., 1966), and its characteristics make it difficult for the prevention and treatment of the C. panzhihuaensis stem rot disease.

Stem rot diseases in Cycas szechuanensis and C.ycas shiwandashanica were reported in 2002, with the highest incidence reaching 25\%. And F. solani (Mart.) App.et Woll. was identified to be the pathogen $(\mathrm{N}$. Li, 2002). Therefore, F. solani is a pathogen causing stem rot diseases in Cycads.

The characteristics of the C. panzhihuaensis stem rot disease revealed in our preliminary investigation showed that the disease was not epidemic and did not strike many plants. However, each C. panzhihuaensis, as a Grade 1 National Protected Plant, is invaluable and should be well protected. Based on the results of this research, further studies about the prevention and treatment of this disease will be carried out.

\section{References}

[1] Fu M Z, Xu Z H, Huang Z Q. 2005.Summary of the known diseases and insect pests of cycadaceae in china and their conctro[J].Journal of southwest forestry College,25(2):44-52.

[2] Наo Y Q, Mo X, Yu Z X, Yang $\mathrm{Y}$ Q.2011.Florogeographical Analysis of Spermatophytes in cycas pauzhihuaeusis Assemblage[J].Journal of Sichuan Forestry Science and Technology,32(6): 28-33.
[3] H. L. Barnett, B. B. Hunter.1960.Illustrated Genera of Imperfect Fungi[M]. Shen C Y,1977.Beijing,Science Press.

[4] Li N, Lin Y E, Hu G X, Liu Q F, Deng M R,Wang X R, 2002. Pathogen identification and symptom development of stem-bulb rotten disease in Cycas[J].Forest Pest and Disease,21( 6):8-10.

[5] Li X X, Xiao Z J, Xu A B, Xu G H. 2014.Identification of leaf blight pathogen on cycas revoluta thunb[J].Chinese journal of tropical crops, 35(10):20662070.

[6] Li Y, Wu Z Q, Jing Y B, Pang J, Zhao Y H, Li C P, Zeng Y M, Wu J R.2013.Identification of Damping-off Pathogen on Betula alnoides[J].Journal of West China Forestry Science,32(6): 28-33.

[7] Lin L W, Li Z X, He X W, Huang S H.2003.Pathogenic identification of root rot disease of Pueraria thomsonii[J].Ecology and Environment,12(4):516-517.

[8] Luo J J, Liu Q G, He Y B, Li Z B, Wei C D.2012.Identification of the pathogen causing pineapple heart-rot disease[J].Guangdong Agricultural Sciences, 39(13): 90-92.

[9] Shen L T,Li P, Wang X G, Zhang M. 2012.Biological Characteristics of the Pathogen of Root Rot of Yam[J]. Journal of Sichuan Agricultural University, 30(3):313318.

[10]Wei J C.1979. Fungi Identification Manual[M].Shanghai,Shanghai Scientific and Technical Publishers.

[11]Wu Y K , Zhu X E, Ou G L , Yu J Y, Sun J C.2011.Biological characteristics of Fusarium solani causing root rot of Jatropha curcas L.[J].Guangdong Agricultural Sciences, 38(24): 62-65.

[12] Yang L Y, Huang H P, Tang F R, Hu M J, Zhang S Q, Huang J S. 2006. Rapid molecular identification and detection of Colletotrichum musae with species-

[13] specific primers based on the internal transcribed spacer(ITS)region[J].Acta

Phytopathologica Sinica,36(3): 219-225.

[14]Zhou L, Yan S Y, Fu L K,Cheng S Z.1981.Two new species of Cycas from Sichuan[J].Acta Phytotaxonomica Sinica,19(3): 335-338.

[15] Bateman D.F. 1966.Hydrolytic and trails-eliminative degradation of pectic substances by extracellular enzymes of Fusarium solani f. phaseoli.[J] Phytopathology.56(2):238-244. 